\title{
Performance Evaluation of the Dependable Properties of a Body Area Wireless Sensor Network
}

\author{
Venki Balasubramanian and Andrew Stranieri \\ Centre for Informatics and Applied Optimisation \\ School of Science, Information Technology and Engineering \\ University of Ballarat, Ballarat, Australia \\ \{v.balasubramanian, a.stranieri\}@ballarat.edu.au
}

\begin{abstract}
Body Area Wireless Sensor Networks (BAWSNs) are self-organizing networks capable of monitoring health intrinsic data of a patient. BAWSNs extended with a healthcare application can be used to perform medical assessments by remotely monitoring patients. The accuracy of medical assessments fundamentally depends on the correctness of the data received from the BAWSN. However, data errors may arise at the sensor or during transmission across the wireless sensor network. Therefore, it is imperative to measure the health intrinsic data of a patient precisely. The formulated measurable properties in our work precisely measure the performance of the BAWSN in a remote Healthcare Monitoring Application (HMA). In this paper, we collated various performances using the measurable properties in our real-time test-bed and presented a comprehensive evaluation of these properties in a BAWSN.
\end{abstract}

Key words: BAWSN, ehealth, reliability, availability, critical time, remote healthcare monitoring application

\section{INTRODUCTION}

In Australia, the number of people over 80 years old has increased from 260,000 (recorded in 1981) to 560,000 (recorded in 2001) and, it is expected to surpass 790,000 by 2021 [1]. As the home of one of world's largest population, the population of India will continue to grow through 2050. By 2030 , it is estimated that $45.4 \%$ of the Indian population would be older adults [2]. Furthermore, in India, the sixty-plus age group will grow more than threefold from $8.4 \%$ to $22.6 \%$ over fifty years, while those aged eighty and older will expand nearly fourfold from $0.8 \%$ to $3 \%$ [2]. This trend is not unique to Australia and India, rather it is a global issue because it has been estimated that the global population of 65 years old and above will double from 357 million (recorded in 1990) to 761 million by 2025 [3].

In addition to the growth in the ageing population, there has been an explosion in the prevalence of chronic conditions. Chronic conditions including diabetes and cardiovascular disease cannot be cured and must be closely monitored for the emergence of complications. However, many chronic diseases show notable symptoms of the disease or complications only in their later stages [4-6]. Continuous monitoring of a health condition is a proven method for early detection of many conditions [4]. However, many developed and developing nations already plagued by the high-rising costs of healthcare owing to the large number of chronic patients are further challenged to resource regular monitoring. Therefore, in-house remote healthcare monitoring applications provide an alternative by delivering healthcare to a dispersed population having a variety of diseases requiring regular monitoring and care. This is especially true in suburban or rural areas where expert doctors, nurses, electronic equipment and other infrastructures are often inadequate or difficult to access in time.

One of the major challenges in remote healthcare monitoring application (rHMA) involves conducting medical assessments based on remotely sensed patient's health data. The accuracy of the health data such as sensed time, unaltered data content and identity of the person should be intact to avoid false diagnoses. However, ensuring data is accurate is difficult in a Body Area Wireless Sensor Networks (BAWSN) because sensors are not always reliable or accurate and data transmission over a wireless network is also not perfect. We can expect BAWSN's to be increasingly accurate with advances in sensor and wireless technologies; however an innovation that will always be useful is a metric that provides an estimate of how accurate a BAWSN can be expected to be. A set of dependable properties for a BAWSN were proposed and formulated in our work in [7]. In the current work, we show how these properties can be used to estimate the accuracy of a BAWSN in a real-time test-bed.

The paper is arranged in the following sections. Section II will describe a remote healthcare monitoring application and our background work. In Section III, we briefly describe properties that make a BAWSN dependable. Section IV will give the details of the experimental setup, while the performance of the operational properties will be given in Section V. Finally, Section VI will conclude this paper.

\section{REMOTE HEALTHCARE MONITORING APPLICATION}

The rHMA is an application system made up of two related applications, the healthcare application (HA) and the BAWSN, the monitoring application component:

1. The BAWSN consists of state-of-the-art self-organizing wireless sensors capable of sensing vital health data of a patient, a base station, which aggregates the health data from the sensors, and a local processing unit (LPU), which can be a smart phone or a tablet.

2. The HA should be considered as a system that uses information technology for collecting, maintaining, analysing and disseminating the patient's health data for 
clinical needs. Although, maintaining and disseminating the patient's data are achieved through the use of various technologies, to measure and collect the health-related data of the patient, the HA uses a manual/traditional method that requires the patient's attendance at the hospital.

Our real-time test-bed Active Care Loop Framework (ACLF) is an rHMA is designed and built using various wireless technologies and the Internet as the data transfer medium. It consists of the Assistive Maternity Care as it's HA and the BAWSN, the monitoring application component.



Fig. 1. Active Care Loop Framework

The AMC application enables practitioners (such as doctors and midwives) and pregnant women to access health records from beyond the physical location of a hospital zone not only through desktop computers, but also through smartphones as shown in Fig. 1. In the AMC application, a pregnant woman enters her blood pressure (BP) value using a smartphone or desktop regularly at home or at work, for instance, to allow her health care providers to monitor her condition. Depending on the gravity of updated data, the database notifies the AMC application that would then automatically send an alert message with specific information on the patient to the care staff, either as an SMS or an email [7].

The AMC application described above requires the manual entry of the health data by the pregnant women. To automate the entry of the health data, the AMC application is extended with a BAWSN to form the ACLF. For instance, in the AMC, it is inconvenient for a pregnant woman to continuously enter health data, such as the BP, heart rate and glucose levels, for monitoring purposes. Therefore, a BAWSN is used to sense the required health data at regular time intervals. The distinct functions of a BAWSN is to authenticate the patient for continuous monitoring, to sense the health data from the patient, to pre-process the health data of the patient for sending any alert messages in the case of an emergency and to send the preprocessed data to the HA for further medical diagnoses. It is evident that the rHMA depends heavily on its monitoring component, the BAWSN, for the continuous generation of the health data. The main focus of our work is to evaluate the performance of the BAWSN, while the reliability and availability of the AMC application is outside the scope of this paper.

\section{THE DEPENDABLE PROPERTIES}

To build a BAWSN, the underlying wireless sensors network (WSN) should be extended with an LPU, that is, a smartphone, for further processing of the data. In general, for any underlying networks, one might use Quality of Service (QoS) as a property to evaluate WSN performance. However, QoS is a broad term used to denote the level of predictability and manageability of the services supplied by one or more service providers. Moreover, the QoS refers to resource reservation control mechanisms rather than the achieved service quality.

In computer networks, the QoS is the ability to provide different priorities to different applications, users or network data flows or to guarantee a certain level of performance for a data flow [9]. The QoS is more applicable for the underlying network in a given application system. Although the underlying wireless networks form the foundation for a BAWSN, the BAWSN itself forms an integral application component in an rHMA system. In addition, the functionalities in a BAWSN are also well defined and termed as critical in an rHMA system because it has the onus of collecting health data intrinsic to the patient. Therefore, providing different priorities to different applications may not be appropriate.

Further, the properties that define QoS for a BAWSN [9] do not emphasise the security aspects that are defined as important for the BAWSN [10- 13]. In addition, the construction and the maintenance of components are less explored areas, even though they are vital for achieving accurate collection and transmission of data and fault detection. The studies in $[11,12]$ also show that the QoS in a BAWSN is not scalable in terms of power consumption and cannot be used as a single solution for all kinds of BAWSN applications. Based on these shortcomings in using only the QoS for the performance evaluation, the properties that define the dependability of the component are considered. Highly sought properties for WSN, the node power and node destruction/repair, is encompassed by the availability property [14-16] from the dependability property list. Therefore, the dependability properties comprise: reliability, availability, security, maintainability and safety. These form an effective set for evaluating the performance of a BAWSN.

Apparently, not every property of dependability can be measured numerically, because certain properties such as maintainability and safety can only be realized by assessing their flexibility in the design during development and their adaptability to the environment during its real-time deployment. However, properties such as availability, reliability and security can be quantified. In our research work [7] the reliability and availability properties were formulated based on derived time-specific parameters. The time-specific parameters along with the critical time are described in the following sub-section.

\section{A. Time-critical applications}

The drive for the rHMA is to provide for continuous remote monitoring of the health condition of the patient. For this to happen, the sensors in the monitoring component of the BAWSN should continuously send data to the HA through the LPU. To provide continuous health data, the BAWSN relies on the collective data provided by all the source sensor nodes and not on any individual data, because medical diagnosis based on 
health data is rarely based on any one specific parameter [1719]. In addition, the arrival time of the data should be within the expected time $\left(\varepsilon \mathrm{t}_{\mathrm{m}}\right)$ because any arrival of health data outside the expected time cannot be assumed to indicate the actual health condition of the patient. For example, a high blood pressure and a high heart rate when a person is at rest is a clinical incident and should be taken care of immediately; whereas, the same condition for a person during substantial movement may not be a clinical incident. It should be noted that the arrival time of health data, both the blood pressure and a heart rate is necessary for medical diagnosis. Therefore, in our work [7], we defined and implemented a novel critical time parameter for the BAWSN operations. Because the BAWSN has stringent timing requirements concerning the arrival data rate from the sensor nodes within the defined critical time $\left(\varepsilon \mathrm{t}_{\mathrm{m}}\right)$, we characterized the rHMA as a time-critical application. Based on the time stringent requirements and to address generic measures such as delay, jitter and packet loss, the following time critical parameters have been selected [7]:

1. The critical lack time $\left(\mathrm{T}_{\mathrm{LACK}}\right)$, is the elapsed time in the application for which the medical diagnosis may not be accurate owing to the delayed packet arrival.

2. The error time $\left(\mathrm{T}_{\mathrm{E}}\right)$, is the defective time in the application caused by erroneous packets.

3. The buffering error time $\left(T_{B}\right)$, is the defective time in the application caused by the loss of packet.

The parameter used in the formulation of reliability and availability properties is given in the following table:

TABLE I. TIME-SPECIFIC PARAMETERS

\begin{tabular}{|c|c|}
\hline$\varepsilon t_{m}$ & $\begin{array}{l}\text { Expected time interval for a BAWSN to receive } \\
\text { data from the sensors. The critical time rate for } \\
\text { the BAWSN application that could be used for } \\
\text { monitoring purpose. }\end{array}$ \\
\hline$n$ & Index for counting sensors in a BAWSN \\
\hline$T_{L A C K}$ & $\begin{array}{l}\text { Critical lack time, elapsed time for which the } \\
\text { application is inoperative owing to delayed packet } \\
\text { arrival }\end{array}$ \\
\hline$T_{E}$ & $\begin{array}{l}\text { Error time, inoperative time of application owing } \\
\text { to erroneous packets }\end{array}$ \\
\hline$T_{B}$ & $\begin{array}{l}\text { Buffering error time, inoperative time of } \\
\text { application owing to loss of the packet in the base } \\
\text { station buffer }\end{array}$ \\
\hline$T_{E}^{S_{n}}$ & Total error time for the sensor $S_{n}$ in a BAWSN \\
\hline$T_{E}^{S_{n}}$ & $\begin{array}{l}\text { Total buffering error time for the sensor } S_{n} \text { in a } \\
\text { BAWSN }\end{array}$ \\
\hline$T_{E B}^{S_{n}}$ & $\begin{array}{l}\text { Total error and buffering error time for the sensor } \\
S_{n} \text { in a BAWSN }\end{array}$ \\
\hline$T_{\text {LACK }}^{S}$ & Total critical lack time in a BAWSN \\
\hline
\end{tabular}

Based on the above time-critical parameters total unreliable time of the sensors and the total unavailable time of the sensors in a BAWSN are given by the below Eq. 1 and Eq. 2 respectively.

$$
\begin{gathered}
T_{U R}^{S}=T_{L A C K}^{S}+T_{U A}^{S}-\sum_{r=1}^{B} R_{L A C K}^{r} \\
T_{U A}^{S}=\frac{1}{2} \sum_{n=1}^{N} T_{E B}^{S}-a \cdot s t_{m}
\end{gathered}
$$

The operational availability of the BAWSN states that a system is highly available if the fraction of its downtime is very small, either because failures are rare or because it can restart very quickly after a failure, while the operational reliability of the BAWSN is defined as seamless delivery of error free health data within the critical time. By using the Eq. 1 and Eq. 2 the operational availability and reliability of the BAWSN is given by Eq. 3 and Eq. 4 respectively.

$$
\begin{gathered}
A_{B A W S N}=1-\frac{T_{U A}^{S}}{t} \\
R_{\text {BAWN }}=1-\frac{T_{U R}^{S}}{t}
\end{gathered}
$$

To show how these properties can be used to evaluate the performance of the BAWSN in real-time is just as important as formulating these properties for the application developer. The following sections describe the experimental facilities and the results are analysed using the time-critical parameters.

\section{EXPERIMENTAL FACILITIES}

The experiment was conducted in the BAWSN, as shown in Fig. 2 . The BAWSN includes heterogeneous components differing not only in processing power and storage capacity, but also having a diverged operating platform. The sensor components consist of four crossbow-technology-based sensor motes [20] that operate using the TinyOS [21]. The end-point is the local processing unit, a smartphone connected with a Stargate gateway board [20], which is used as the base station to interconnect the 802.15.4/ZigBee-based crossbow family motes and the 802.11-based smartphones. A Stargate can be used as a base station only after housing a base sensor mote in the Stargate board. Please note that Fig. 2 shows six mote sensors; however, during our initial experiment to calculate the availability and reliability of the BAWSN, only four sensors were used. To implement and test the proposed security scheme in the BAWSN, six sensors were used in which the sensors 
marked 5 and 6 in Fig. 2 have been programmed with a different identification set. These two sensors are used to test the behaviour of the security scheme in the BAWSN, which is outside the scope of this paper.

The mote sensor and the Stargate (base station) have hardware on/off switches, with the sensors being programmed to transfer data packets every 10 seconds to the base station when the switch in the BAWSN is turned 'on'. The user initiates the continuous monitoring application Obi-MATE using a smartphone. The application starts the protocol that registers the smartphone with the base station. Once the smartphone has been registered, the gathered data at the base station can be fetched through the Stargate gateway. Because the base sensor mote attached to the Stargate has a limited buffer memory for storing the sensed data received from all the sensor nodes, it is essential that data are fetched from the base station before the buffer overflows and causes a loss of data.

Therefore, we have modelled the smartphone to request the Stargate at a specific frequency, $\mathrm{f}_{\mathrm{R}}$ the number of requests sent from the smartphone over a selected time interval should satisfy the following inequality:

$$
f_{R} \prec \frac{N \cdot d}{S-C}
$$

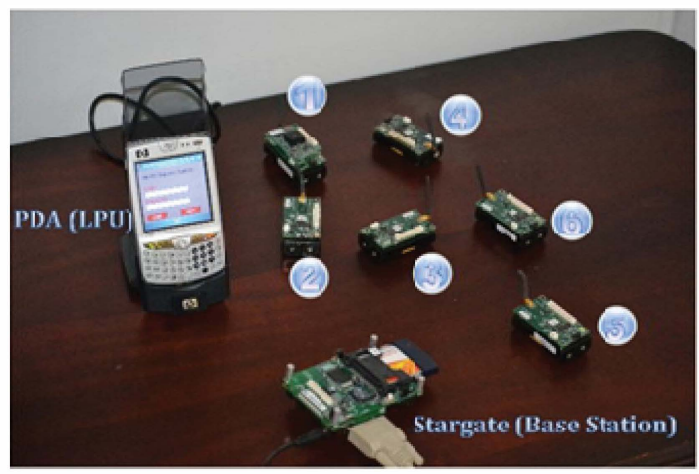

Fig. 2. Experimental setup for the BAWSN

The concept of developing assistive mobile application for the pregnant women and the midwives in ACLF has been given the name of Obi-MATE

Where,

$$
\begin{array}{ll}
\text { - } & \text { S is the size of the maximum base sensor buffer (bytes) } \\
\text { - } & \text { C is the current level of base sensor buffer (bytes) } \\
\text { - } & \text { N is the number of sensors used in a BAWSN } \\
\text { dis the data rate of the sensors (bytes/sec). }
\end{array}
$$

The above inequality means that data must be requested by the end-point (smartphone) before the buffer of the aggregation point (base station) is full: otherwise, it is lost. This inequality is programmed into the smartphone to mitigate the loss of the packets in the base station buffer. After the initial request, the smartphone computes the request time interval $t_{R}\left(1 / f_{R}\right)$ on the fly, at which time the next request is sent, based on the current buffer level $C$, whereas the other factors $S, N$ and $d$ are kept constant for a given BAWSN [7]. Note that the smartphone's request time interval $t_{R}$ can be achieved merely by using the lesser value compared to the programmed time in the sensor less than 10 seconds in our scenario. This proved to be highly inefficient in terms of sending a request to the base station, even if the buffer is empty. Consequently, these inefficient requests considerably reduce the smartphone's battery power. Moreover, the application developer can configure the monitoring application to compute the request time interval based on $N, C$ and $d$ instead of hardcoding the request time interval in the monitoring application, because $t_{R}$ depends on the requirement of the continuous monitoring and the data rate of the sensors used in the BAWSN. The inequality in Eq. 5 enables the endpoint to send a request to the base station just before the buffer is full and empties the buffer. This timely request from the smartphone gradually reduces the base station buffer overflow and also ensures that no data is lost owing to implementation/system faults. The following section presents the results for reliability and availability obtained by conducting the experiments in the real-time test bed [7].

\section{EXPERIMENTAL RESULTS}

The packet size for all the sensor motes was configured to 29 bytes, including the header. During the initial phase, the data packets of the sensors were not encrypted. To study the impact of inequality on the performance of the BAWSN and also to calculate the operational properties, a series of experiments were conducted in our BAWSN under similar conditions with each a duration of 60 minutes. The average result of the five experiments is plotted as shown in Fig. 3. The properties reliability and the availability values in the experiment are calculated for every 10-minute interval within the actual duration of the experiment.

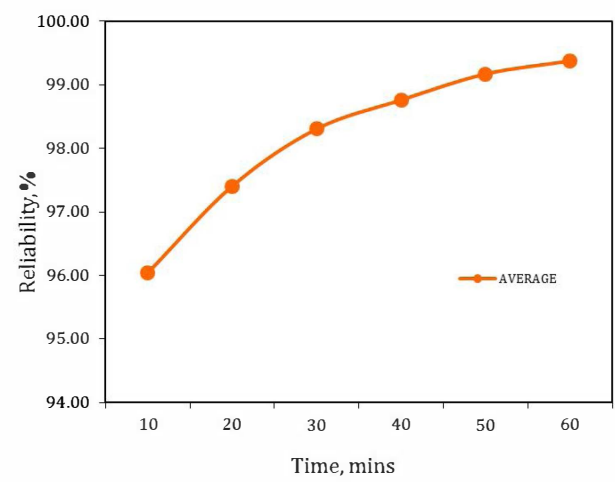

(a)



(b) 


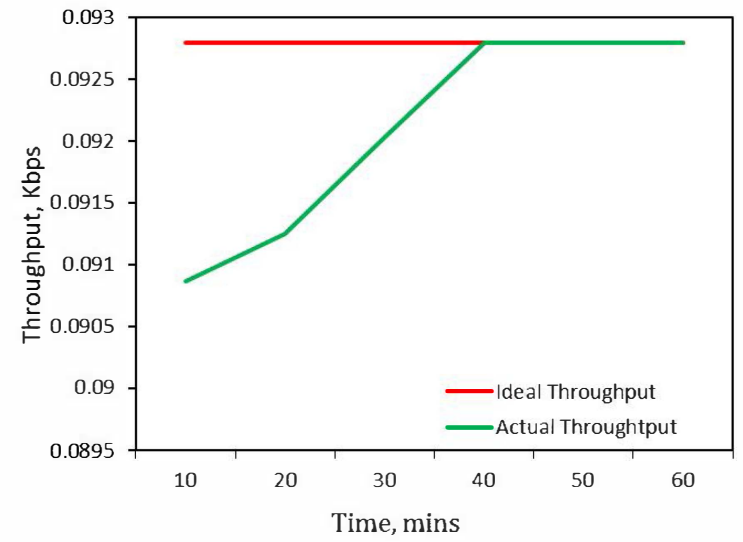

(C)

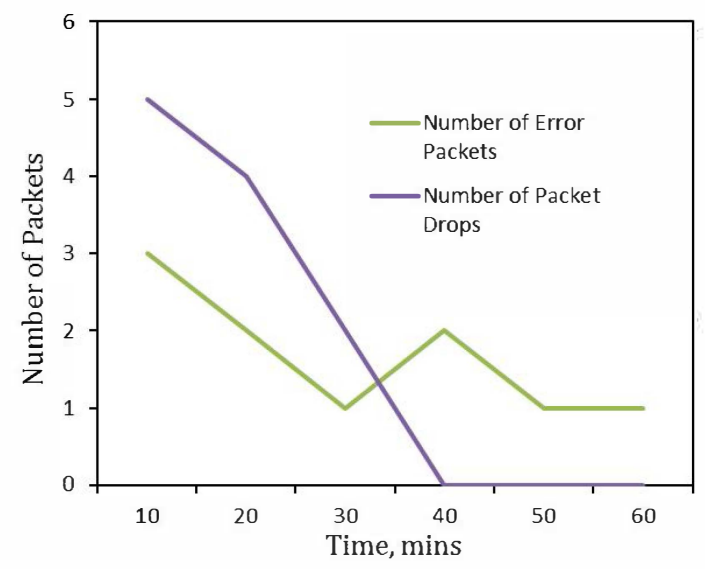

(d)

Fig. 3. Performance of a BAWSN (a) Operational Reliability (b) Operational Availability (c) Operational Throughput (d) Number of error and lost packets during operational time

These experiment results demonstrate a gradual increase in the operational properties throughout the duration of the experiment. The decreased reliability and the availability during the initial interval is caused by the system calculating the critical time during this initial phase and also, the programmed inequity in the smartphone learns about the buffer level only after a few requests, because there were some dropped packets.

Following this, the experiments demonstrate that the loss of the packets in the base station buffer is gradually reduced, thereby mitigating $T_{B}^{S}$. A significant reduction in $T_{L A C K}^{S}$ is also noted owing to the timely retrieval of the packets from the base station. It is noted that, because of the decreased $T_{B}^{S}$. and $T_{L A C K}^{S}$ the operational properties of the BAWSN foster and stabilize with time, because of the negligible $I_{E}^{S}$ and $T_{L A C K}^{S}$ are achieved; the operational properties remain basically unchanged, because a consequential $T_{E}^{S}$ is noted from time to time throughout the experiment. In Fig. 3(a) and (b), the operational properties show very little increase in the latter half of the experiment. The consequential $T_{B}^{S}$ during the experiment is caused by the use of various wireless technologies, such as ZigBee and Wi-Fi. It is evident from the Fig. 3(d), the packet required [22] to establish the actual cause of error packets that can be controlled by the user, so that the reliability and availability of the BAWSN can be improved. On the other hand, the possibility of attaining maximum operational properties is to obtain a negligible $\Delta t_{c}$. This can be achieved by providing a common hardware switch for the sensors, so that all the sensors can be turned 'on' simultaneously. The Fig. 3(c) shows the comparison of an ideal throughput and the actual throughput (from for sensors) for the operational time. The purpose of this graph is to substantiate that the throughput measure in the BAWSN is of a lesser significance to measure its performance. Because, during the initial phase of the experiment, the actual throughput is less than the ideal because of the dropped/lost packets; however, it is in line with an ideal throughput in the later part that demonstrates that system is working flawlessly. But this is not the case, because the occurrence of an error packet is also considered during the throughput measurement. Also, Fig. 3(d) shows the number of dropped packets during the operational time that might be an inappropriate measure for evaluating the performance of the system. Most of the packets drop at the same time instant during the initial part of the experiment, so the system is not performing well only at that time instant, not for that complete interval.

\section{CONCLUSION}

The paper exemplifies the importance of a BAWSN in a remote healthcare monitoring application. The justification for dependable properties for a BAWSN along with the overview about the time-specific parameters used in the dependable properties is also presented. As a major focus the performance evaluation of the operational properties particularly the reliability and the availability of the dependable application with experiment results conducted using the real-time test-bed. Finally, it is important to note that the performance evaluation shown in this paper does not advocate the accuracy of usage in any medical diagnosis.

\section{REFERENCES}

[1] Human Rights and Mental Illness, Available: http://apo.org.au/research/national-inquiry-concerning-humanrights-people-mental-illness, accessed 20 Dec 2013.

[2] The Health Of Aging Populations In China and India, Available: http://content.healthaffairs.org/content/27/4/1052.full, accessed $25 \mathrm{Dec} 2012$

[3] N. Hooyman and H. Kiyak, Social gerontology: A multidisciplinary perspective, 6th ed., Allyn and Bacon, 2002.

[4] K. Kancherla, R. Chilkapatti, S. Mukkamala, J. Cousins, and C. Dorian, "Non Intrusive and Extremely Early Detection of Lung Cancer Using TCPP," Computing in the Global Information Technology, ICCGI '09, Fourth International Multi-Conference, 23-29 Aug. 2009, pp.104-108.

[5] World Health Organization, Available: http://www.who.int/mediacentre/factsheets/fs317/en/index.htm, accessed 24 January 2009.

[6] International Diabetes Federation (IDF), Available: http://www.idf.org, accessed 24 January 2009.

[7] V. Balasubramanian, and D. B. Hoang, "Reliability Measure Model for Assistive Care Loop Framework using Wireless Sensor Networks." International Journal of Healthcare Engineering, vol. 1, no.2,pp. 239-254, 2010. 
[8] Q u a li t y of S e rvi ce, A v a i l a b l e : http://en.wikipedia.org/wiki/Quality_of_service, accessed 24 January 2009.

[9] D. Chen and P. K. Varshney, "Qos support in wireless sensor networks: A survey," in Int. Conference on Wireless Networks (ICWN 2004), CSREAPress, June. 2004.

[10] B. Latre', B. Braem, I. Moerman, C. Blondia, and P. Demeester, "A Survey on Wireless Body Area Networks," Journal of Wireless Network, vol. 17, Issue. 1, pp. 1 -18, January. 2011.

[11] I. F. Akyildiz, W. Su, Y. Sankarasubramaniam, and E. Cayirci, "A survey on sensor networks," IEEE Communications Magazine, vol. 40, no. 8, pp. 102-114, Aug. 2002.

[12] A. Bhargava and M. Zoltowski, "Sensors and wireless communication for medical care," in Database and Expert Systems Applications, 2003, in Proceeding. 14th International Workshop on, Sep. 2003, pp. 956-960.

[13] S. Cherukuri, K. K. Venkatasubramanian, and S. K. S. Gupta, "BioSec: A Biometric Based Approach for Securing Communication in Wireless Networks of Biosensors Implanted in the Human Body," in Proceedings of the 2003 International Conference on Parallel Processing Workshop (ICPPW'03), 6-9 Oct. 2003, pp. 432-439.

[14] L. Xian, "On the Availability of Wireless Sensor Networks," in Proceedings of the 10th IEEE Wireless Communications \& Networking Conference, Las Vegas, USA, 2008, pp. 3081-3086.

[15] P. Sazia, S.K. Dong, and S.P. Jong," Towards Optimal Software Rejuvenation in Wireless Sensor Networks using SelfRegenerative Components," in Proceedings of 2nd International Conference on Information Security and Assurance, Busan, Korea, 2008,pp. 295-300,

[16] P. Sazia, S.K. Dong, and S.P. Jong, "Towards Survivable Sensor Networks using Self-Regenerative Rejuvenation and Reconfiguration," in Proceedings of 1st International Conference on Information Security and Assurance , Manchester, UK, 2007,pp. 546-549.

[17] What is Prostate Cancer, Available : http://www.prostate.org.au/articleLive/pages/What-is-ProstateCancer.html, accessed 03 October 2009.

[18] E. Ergezen, R.W. Hart, R. Philip, and R.M. Lec, "A high frequency thickness shear mode (TSM) sensor for detection of biomarkers for prostate cancer," Frequency Control Symposium, 2008 IEEE International, 19-21 May. 2008, pp. 341-345.

[19] M. Caron, G. Choquet-Kastylevsky, and R. Joubert-Caron, "Cancer Immunomics Using Autoantibody Signatures for Biomarker Discovery," Molecular and Cellular Proteomics, pp. 1115-1122,2007.

[20] Crossbow Technology Inc., Motes, Wearable Sensors, Available : http://www.xbow.com/, accessed 24 January 2010.

[21] TinyOS Embedded Operating System, Available: http://www.tinyos.net, accessed 24 January 2010.

[22] S. Shin, S. Choi, H.S. Park, and W.H. Kwon," Packet error rate analysis of IEEE 802.15.4 under IEEE 802.11b Interference," in Proceedings of the 3rd International Conference on Wired/Wireless Internet Communications, LNCS Springer, Xanthi, Greece, 2005, pp. 279-288. 